\title{
Sildenafil reduces pulmonary hypertension in HF
}

Cyclic GMP (cGMP) release is decreased in patients with heart failure (HF) and elevated pulmonary vascular resistance but can be increased by the phosphodiesterase 5A inhibitor sildenafil, which also reduces pulmonary arterial pressure. These findings, from a study by researchers in the Czech Republic, provide in vivo confirmation of the beneficial effects of phosphodiesterase inhibitors in the treatment of pulmonary hypertension.

Chronic HF can cause changes in the pulmonary vasculature that increase both vascular resistance and pulmonary arterial pressure. In patients with heart failure, pulmonary hypertension confers a poor prognosis, and treatment options for such individuals are limited. "If a normal heart from a donor is transplanted into a patient with $\mathrm{HF}$ and pulmonary hypertension, the new heart suddenly has to pump blood against increased resistance and the graft may fail early after the procedure," explains researcher Vojtech Melenovsky. To gain insight into the underlying causes of pulmonary hypertension, Melenovsky and colleagues examined the role of cGMP on vascular resistance in patients with $\mathrm{HF}$.

Increased vascular resistance was associated with attenuated release of cGMP, but sildenafil restored cGMP release and lowered vascular resistance. These findings highlight the importance of cGMP signaling in the regulation of pulmonary vascular tone. Melenovsky's research group is currently conducting further studies in patients with heart failure to elucidate the reasons for diminished cGMP in pulmonary hypertension.

Sharmini Rajanayagam

Original article Melenovsky, V. et al. Transpulmonary
B-type natriuretic peptide uptake and cyclic guanosine
monophosphate release in heart failure and pulmonary
hypertension. J.Am. Coll. Cardiol. 54, 595-600 (2009). 